(C) 1996 IEEE. Personal use of this material is permitted. However, permission to reprint/republish this material for advertising or promotional purposes or for creating new collective works for resale or redistribution to servers or lists, or to reuse any copyrighted component of this work in other works must be obtained from the IEEE.

\title{
Bunched Beam Cooling for the Fermilab Tevatron
}

\author{
Ralph J. Pasquinelli, Fermilab*, Batavia, IL. 60510 USA
}

\begin{abstract}
Fermilab has been working on bunched beam transverse stochastic cooling in the Tevatron since 1990. In that time much progress has been made in understanding the difficulties of making such a system work with reasonable cooling times. Problems with common mode rejection of longitudinal signals, wide band $\mathrm{GHz}$ signal transmission using fiber optics, and specialized optical techniques of recursive notch filters have been researched and employed. Specialized planar loop pickup and kicker arrays were developed. Signal suppression has been observed. Efforts are underway to eliminate the coherent signals that dominate the Schottky spectrum.
\end{abstract}

\section{INTRODUCTION}

Emitance blow up in the Tevatron has been a cause of decreased integrated luminosity during the history of collider runs. The motivation for using stochastic cooling to reduce emitances and increase integrated luminosity has been reported earlier. ${ }^{l}$ The success of stochastic cooling for DC coasting beams has been proven for over a decade. To date, limited stochastic cooling success has been achieved with bunched beams. ${ }^{2}$ The cooling of high energy beam ( $900 \mathrm{GeV}$ in the Tevatron) has not yet been successful. Four cooling systems have been designed in the 4-8 GHz band, one each for horizontal and vertical proton and antiproton beams. The intention of this paper is to present a progress report and current status of the systems at Fermilab.

\section{HARDWARE}

\section{A. Pickups and Kickers}

When the bunched beam cooling project started in 1990, it was clear that a new type of pickup electrode would be beneficial. The three dimensional designs that have been in use in the Antiproton Source did not have the mechanical tolerances required for this project. A new planar loop array was designed and built. ${ }^{3}$ The necessity of high common mode signal rejection required mechanical tolerances of the arrays to several mils. The arrays used for the current systems are a second generation planar array that no longer requires the use of via holes on the circuit card to connect the loops to the combiner board. The new design has been used successfully not only for this project, but for all cooling upgrades at 1-2 GHz, 2-4 GHz and 4-8 $\mathrm{GHz}$ in the Fermilab Antiproton Source.

Variation in beam size during acceleration necessitated the design of plunging arrays to avoid aperture restriction. Initial tests using a connectorized microwave hybrid for subtracting the lab test signals indicated that the reproducibility of connections was not satisfactory for

\footnotetext{
*Operated by Universities Research Association under contract to the United States Department of Energy.
}

optimal common mode rejection. It was decided to design the arrays on a single circuit card that would also include the differencing hybrid. (figure 1) The hybrid is a patented design ${ }^{4}$ developed at Fermilab that provides better than 0.2 $\mathrm{dB}$ amplitude balance and 2 degrees phase balance across the $4-8 \mathrm{GHz}$ band. When signals are properly timed, this hybrid provides $40 \mathrm{~dB}$ minimum of common mode rejection in the difference output port. An added feature to improve common mode rejection is the use of inchworm motors (piezo electric) on one half of the array. These motors have the ability to move one array longitudinally with respect to the other by approximately $0.25 \mathrm{inch}$. This is in effect an adjustable delay that allows for precision timing of the signals prior to the subtraction in the hybrid.

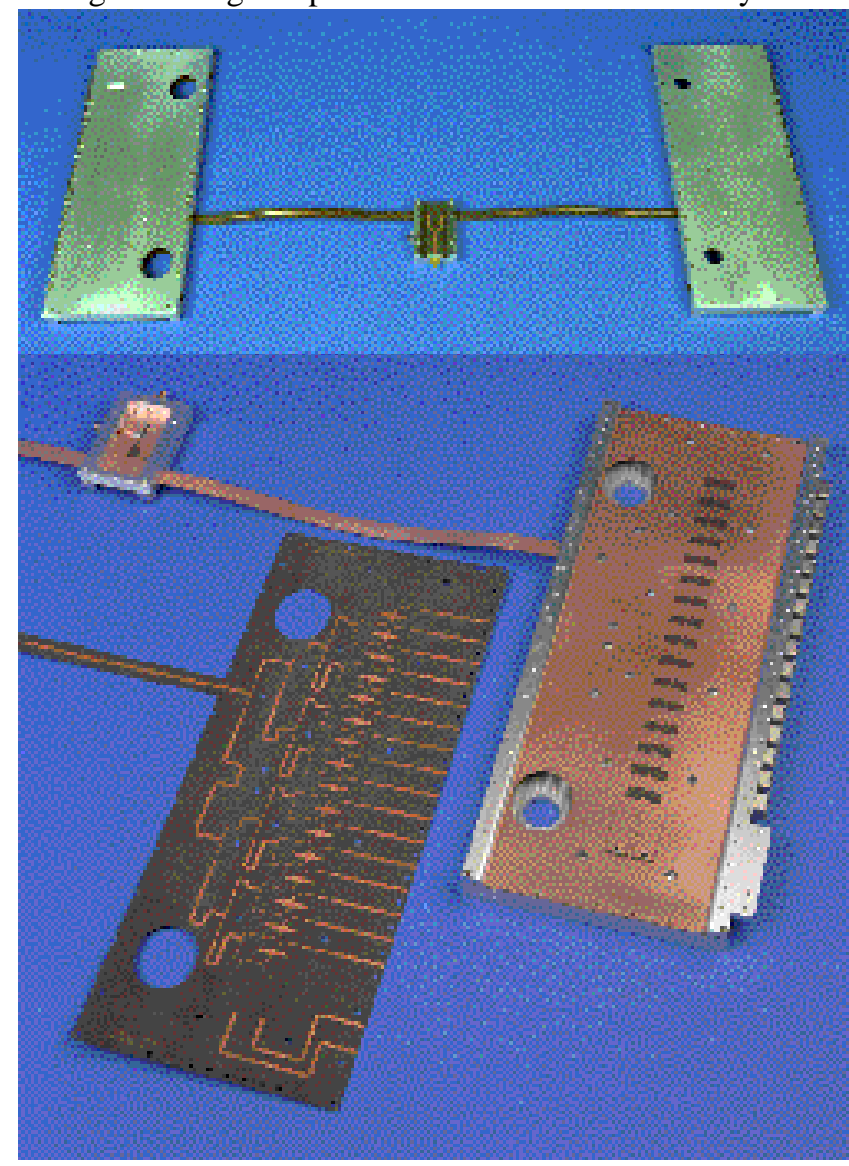

Figure 1: Teflon circuit board with planar 4-8 GHz pickup/kicker arrays. Differencing hybrid is part of circuit board making a connectorless array to hybrid assembly. Shown here laid out flat, the two sides of the array are folded to meet each other in a horizontal or vertical plane.

\section{B. Gating}

Early in the project it was discovered that gating of the feedback system was imperative. The location of the pickups and kickers in the TEVATRON are approximately 100 meters apart. The evanescent modes propagating down the beam pipe were of sufficient amplitude to cause a system oscillation. The gain of the cooling system is in 
excess of $150 \mathrm{~dB}$. Gating interrupts the feedback path of the signal long enough to suppress the instability. An added benefit of the gating is that it improves the signal to noise ratio of signals for diagnostic monitoring. Fast PIN switches controlled by the Tevatron clock system provide the gating in the microwave feed back electronics. Rise times of 10 nanoseconds are typical. Special microwave cutoff devices were also added between the pickups and kickers in order to suppress modes in the beam pipe.

As the beam is only under the kicker for a short portion of the 21 microsecond revolution period, gating also reduces the duty cycle of power delivered to the kickers. This is important in reducing dissipated heat in the structure and minimizes cooling requirements.

\section{Signal Transmission}

The beam transit time of the Tevatron is 20.958595 microseconds. Due to limited space for locating the pickup and kickers in the lattice and the requirement for an odd multiple of 90 degree phase advance for betatron cooling, the delay is in excess of one turn. In order to keep the system phasing correct for cooling, time delay must be kept better than 5 picoseconds out of 21 microseconds. This corresponds to a phase error of 15 degrees at $8 \mathrm{GHz}$, the top frequency of the cooling bandwidth.

\section{s21 Log Mag $1 \mathrm{~dB} /$ Div Ref $-43 \mathrm{~dB}$}

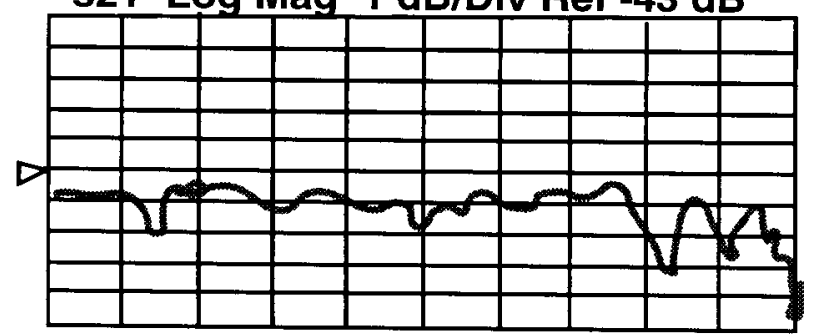

s21 Phase $10 \mathrm{deg} /$ Div Ref $-30 \mathrm{deg}$

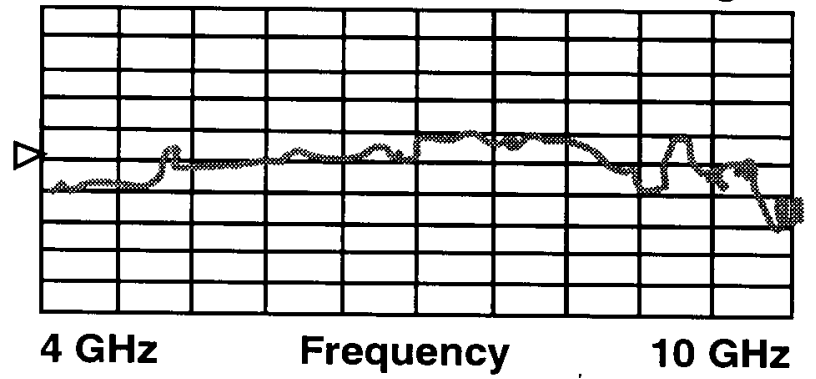

Figure 2: Response of Ortel Fiber Optic Transmission link 4-10 GHz with $6 \mathrm{~km}$ (31.613 usec) of single mode fiber.

Due to the long delay, coaxial cables would not be practical as the insertion loss for such a cable would exceed $1400 \mathrm{~dB}$. The dispersion of such a transmission line would account for many 360 degree phase rotations. This is clearly the regime where optical fiber techniques are mandated. Broad band optical links ${ }^{5}$ have been available and used previously for stochastic cooling notch filters. 6 These links provide the required bandwidth with a flat gain and phase profile (figure 2). The problem still exists that the delay tolerance is stringent. Normal fiber optic cable has a temperature coefficient of $30 \mathrm{ppm}$ per degree
Centigrade. An optical fiber produced by Sumitomo of Japan $^{7}$ is manufactured by depositing a liquid crystal polymer film on the outside cladding of the fiber. The effect is temperature compensation of the fiber as shown in figure 3. This 4.2 kilometer fiber is oven stabilized to better than 0.1 degree Celsius. Delay stabilization to better that a few picoseconds has been achieved. Unfortunately, the Sumitomo corporation is halting production of this fiber due to lack of demand.

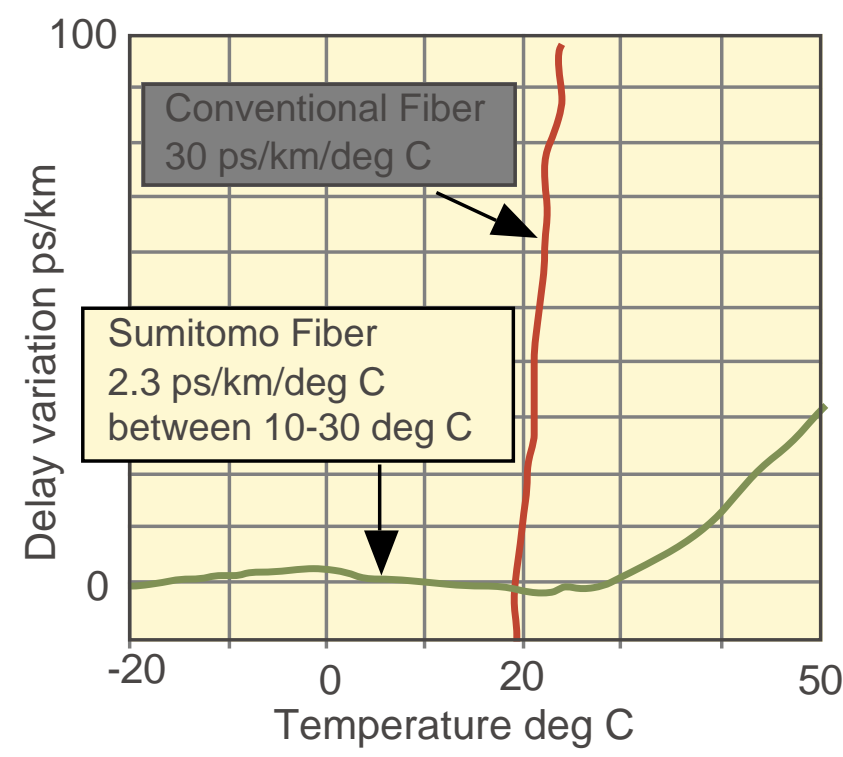

Figure 3: Temperature coefficient of Sumitomo vs. standard single mode optical fiber.

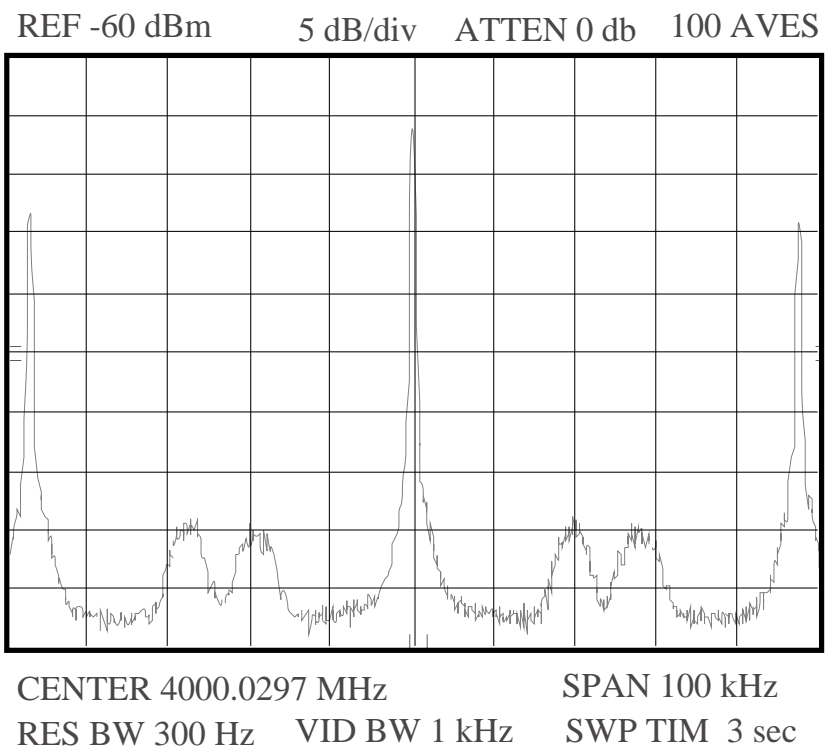

Figure 4: Schottky signal spectrum with gating showing the ratio of coherent to Schottky signal. Intensity is $3.3 \times 10^{10}$ per bunch at $900 \mathrm{GeV}$. 


\section{Notch Filter}

Shortly after the first observation of Schottky signals, it became clear that the longitudinal coherent portion of the spectrum would overpower the transverse signal, figure 4 .

\section{IIR Notch filter Block Diagram}

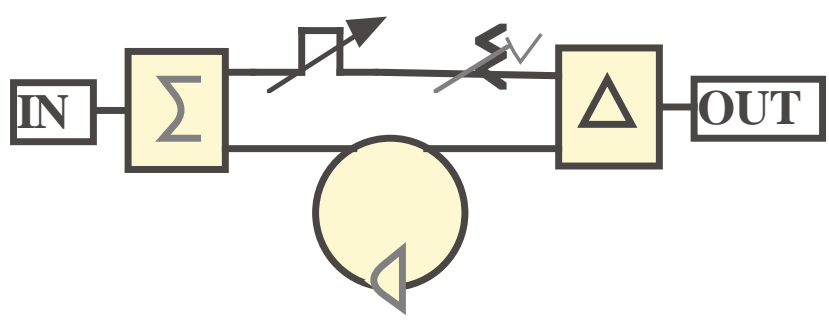

\section{Optical Storage Ring}

Figure 5. Block diagram of IIR optical notch filter.

It was decided that a recursive notch filter needed to be developed. $^{8}$ (figure 5) The width of the Schottky signals occupy a large percentage of the $47 \mathrm{KHz}$ spacing between the revolution harmonics. In order for stochastic cooling to operate, the feedback phase of the system gain would have to be better than 45 degrees across the band, the $3 \mathrm{~dB}$ point. A normal correlator notch filter has a phase excursion of 180 degrees between revolution lines with an additional 180 phase jump at the notch. If such a filter were to be used, the heating and cooling would offset each other with no effective cooling resulting. A new type of filter was developed that employs an optical storage ring. The intent is to produce an infinite impulse response filter (IIR) that can have a "brick wall" amplitude response. (figure 6) This filter is also recursive with a 360 degree phase rotation each revolution harmonic, but due to the infinite impulse response of the storage ring, 340 of the 360 degree phase rotation takes place within $4 \mathrm{KHz}$ of the notch repetition bandwidth of $47 \mathrm{KHz}$.

\section{Amplitude 5 dB/Div}

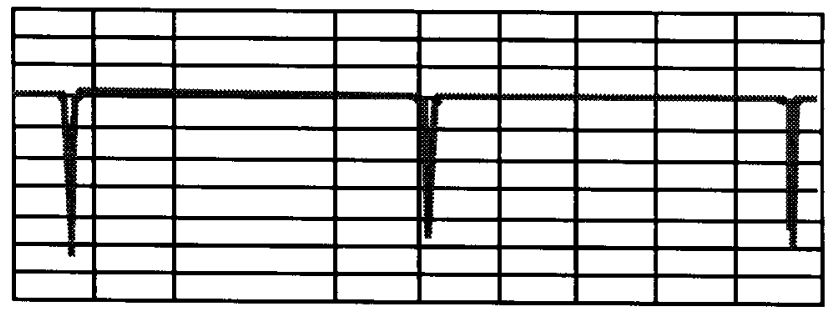

Phase 5 deg/Div

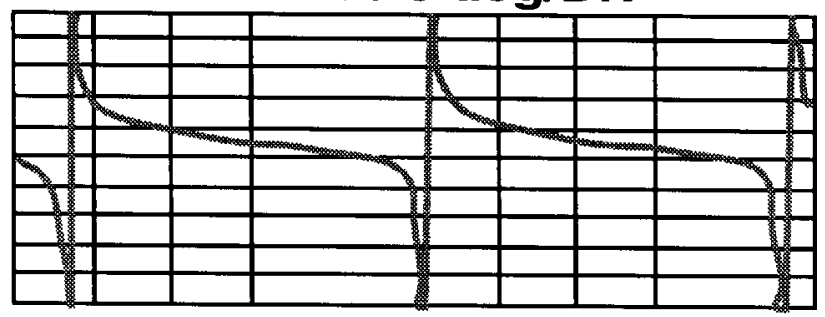

Figure 6. Response of IIR optical storage ring notch filter amplitude (top), phase (bottom). Center freq. $5 \mathrm{GHz}$

This filter is implemented with a fiber optic link similar to the transmission link described earlier. It was very important to use a Fabry Perot type laser transmitter as opposed to a distributed feedback laser(DFB). When the filter was constructed using a DFB, the long coherence length of the laser created an interferometer in the storage ring. A Fabry Perot laser has a short enough coherence length to avoid this problem. The loss in single mode fiber optics is very low, typically less than $0.35 \mathrm{~dB}$ per kilometer. The delay for the optical storage ring must equal the transit time of the particles in the ring.

The resulting 4.2 kilometer fiber along with couplers and other required connections exceeds $2 \mathrm{~dB}$ of optical insertion loss. A source of optical gain is required to make up the loss. Amplifiers that provide gain completely in the optical domain have recently become available in the marketplace. Their development has been spurred by the advancement of fiber optic communications and the desire to minimize the required number of repeaters for transoceanic communications links. Two main types of amplifier are available commercially, laser type solid state amplifiers at 1310 nanometers ${ }^{9}$ and erbium doped fiber amplifiers at 1550 nanometers. Due to the fact that the broad band optical links operate at 1310 nanometers, the choice was limited to the solid state type. Gains of the order of 10 to $15 \mathrm{~dB}$ are typical. The erbium amplifiers have better gain and noise characteristics. The solid state amplifier is basically a laser below threshold and is hence a source of noise that varies with bias current. (figure 7) A very important consideration is the use of a polarization insensitive amplifier. The slightest variation in temperature or mechanical position of single mode fiber will cause polarization rotation. If an amplifier has variable gain as a function of polarization, a unity gain storage ring would be impossible to build. The Q or sharpness of the shape factor of the filter can be adjusted by varying the bias current of the amplifier figure 8 .

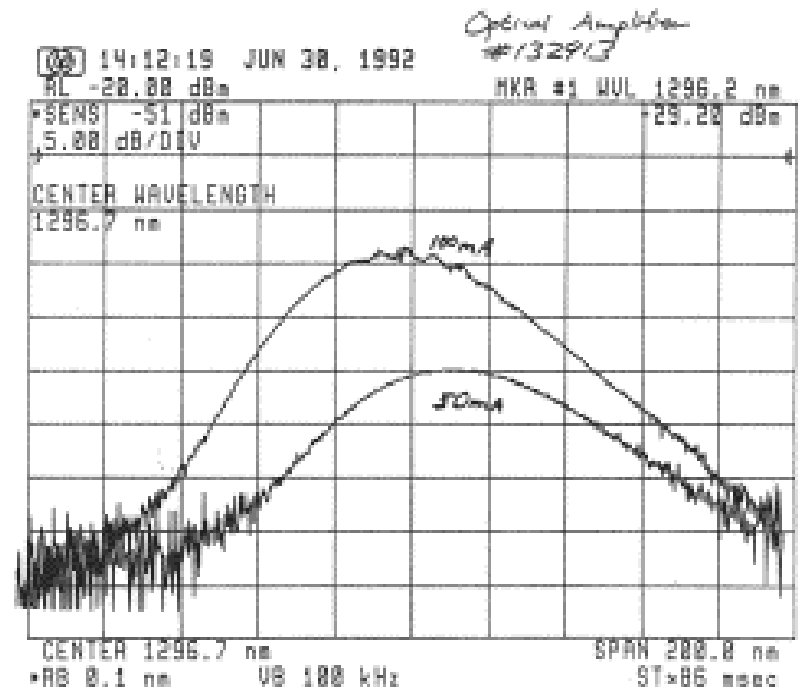

Figure 7: Solid-state optical amplifier optical noise spectrum vs. bias current. Top bias @ 100 mA, bottom @ $50 \mathrm{~mA}$ 
One additional subtlety is the delay of the optical storage ring must be precisely the revolution frequency of the Tevatron at flattop to better than a picosecond Because the signal path is totally optical, a delay mechanism had to be developed that could optically vary the delay. An optical "trombone" using a piezo electric cylinder with single mode fiber wrapped around it would provide a voltage variable optical delay. Such a trombone was designed and built with a $15 \mathrm{~cm}$ diameter piezo cylinder $^{10}$ and provides about 10 picoseconds of delay variation per kilovolt of applied voltage. Because of the difficulty of cutting a 4 plus kilometer fiber to the length accuracy of fractions of a millimeter, regular single mode fiber was used. As described earlier, the temperature coefficient of regular fiber allows for temperature control of the bulk delay time. An oven with feedback is used to tune the coarse length of the fiber (this length fiber has a temp coefficient of 119 picoseconds per degree C) while the piezo trombone does the fine adjustment.

\section{Optical Storage Ring Q vs Gain}

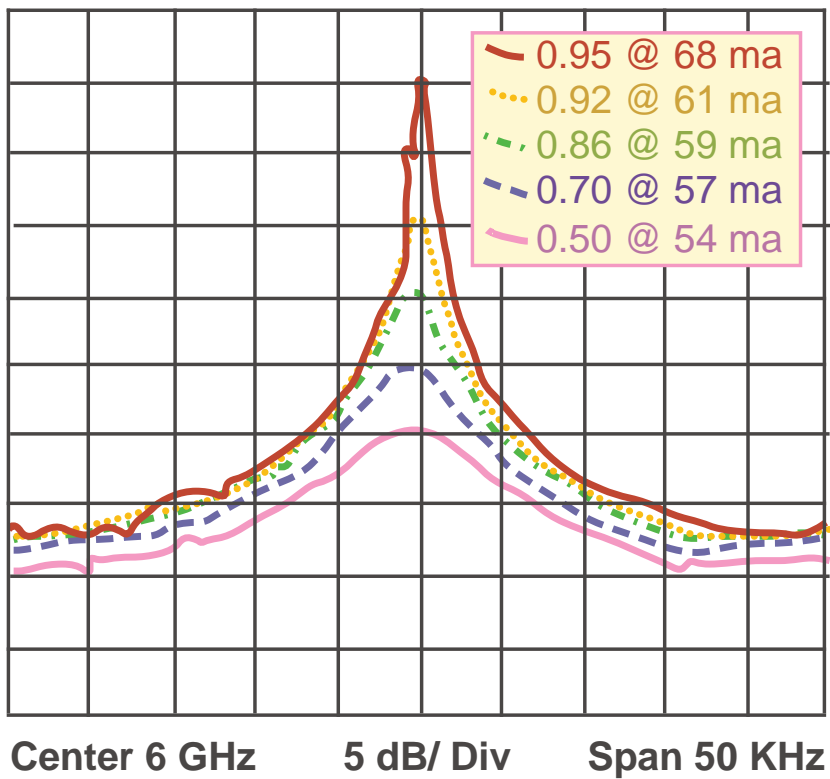

Figure 8: Q or sharpness of response of optical storage ring as a function of optical amplifier bias current. Unity gain is unstable. Gain variation for above curves was 9-12 dB.

\section{EXPERIMENTAL RESULTS}

If one were to examine the bunch structure of the 900 $\mathrm{GeV}$ beam, it would appear that the 2-4 nanosecond bunch length should not contain any frequency components above $2 \mathrm{GHz}$. This is unfortunately not the case. It is believed that the coherent signal is a result of micro bunch microwave structure of the beam. Efforts of reducing the signal via varying the RF voltage were unrewarded as a minimum of RF voltage is required to keep the beam bunched.

The large coherent spikes of the revolution harmonics account for about $99 \%$ of the kicker power. Attempts to reduce this line are the function of the notch filter. The notch filter has a dynamic range of approximately $50 \mathrm{~dB}$ which is adequate. Unfortunately, the filter must be put in the electronic feedback as early as possible to avoid saturation of amplifiers downstream. The signal is pulsed due to the fact that the beam is bunched. The peak power of the signal causes saturation of amplifiers very early in the gain chain. The earliest in the gain chain that it can be located is right after the first preamplifier. At this stage, the signal to noise ratio of the beam signal does not exceed that of the filter itself due to the noise of the optical amplifier. (figure 9) A lower noise source of optical amplification would be required such as erbium, but then there is the incompatibility of the transmission link.

We have abandoned the use of the filter on the front end and have successfully measured transverse transfer functions. (figure 10) The system was timed and signal suppression was observed for the proton vertical plane. (figure 11) It is difficult to estimate the cooling time of such a system as further investigation of signal suppression across the band is necessary. The original requirement of a 20 hour cooling time has not been realized.

The coherent longitudinal spectral lines have also been observed at the ACOL at CERN. ${ }^{11}$ Successful attempts at damping the line using a stochastic cooling system configured as a damper have been successful on the 3.6 $\mathrm{GeV}$ beam at CERN. Attempts to measure a longitudinal transfer function of the $900 \mathrm{GeV}$ Beam in the Tevatron have not been possible to date. The amount of volts necessary to "tickle" the very stiff $900 \mathrm{GeV}$ beam is beyond the capability of installed TWT power.

Ref lev $-80 \mathrm{dBm}, 5 \mathrm{~dB} / \mathrm{div}$

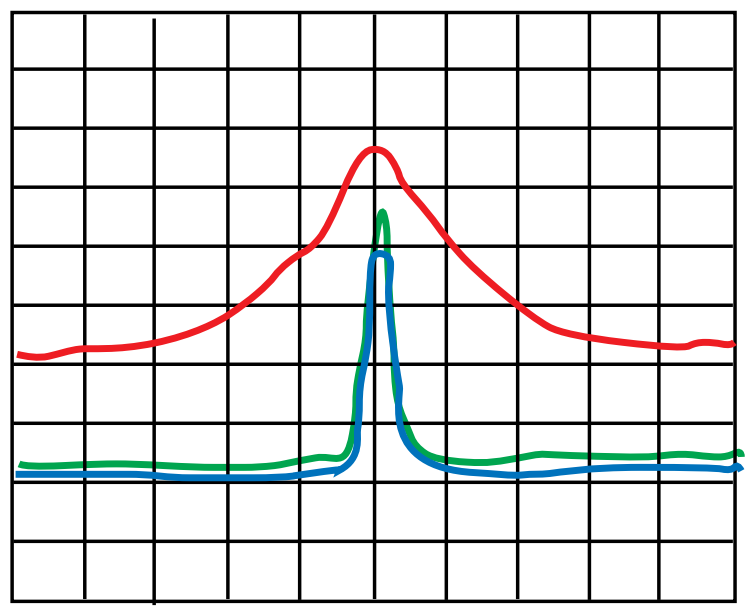

Cen Freq $=4 \mathrm{GHz}$, Span $=50 \mathrm{KHz}, \mathrm{RB}=1 \mathrm{KHz}$

Figure 9: Top trace Schottky output of notch filter, spectrum dominated by optical amplifier noise resulting in microwave noise. Middle trace, coherent signal output of notch filter with optical leg turned off. Bottom trace coherent beam signal input to filter.

The end of the current collider run will be sometime later in the summer of 1995 . The current initial luminosity record for a single store is $2.3 \times 10^{31}$. The original estimates for cooling performance were not based on the beam parameters of present operation which are more intense by about a factor of four. The time remaining before the fixed target physics run begins is limited. Due 
to different Tevatron operating tunes during fixed target, major hardware changes would be required to continue bunched beam cooling experiments. That coupled with lack of extended stores during fixed target would severely cripple any progress. It is imperative to complete our work in the next few months.

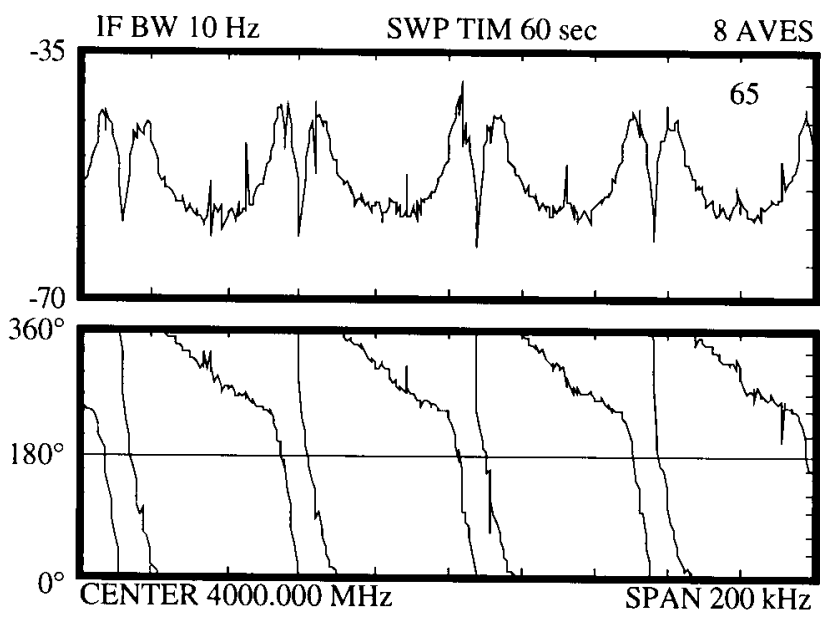

Figure 10: Open loop transfer function measurement. Top, amplitude, showing betatron sidebands. Bottom, phase response. Intensity was $3.1 \times 10^{10}$.

As it turns out, a new storage ring is being proposed within the Main Injector tunnel for recycling and storing antiprotons. 12 If bunched beam cooling proves to be inadequate in the Tevatron, all the hardware can easily be transferred to the new storage ring for coasting beam stochastic cooling.

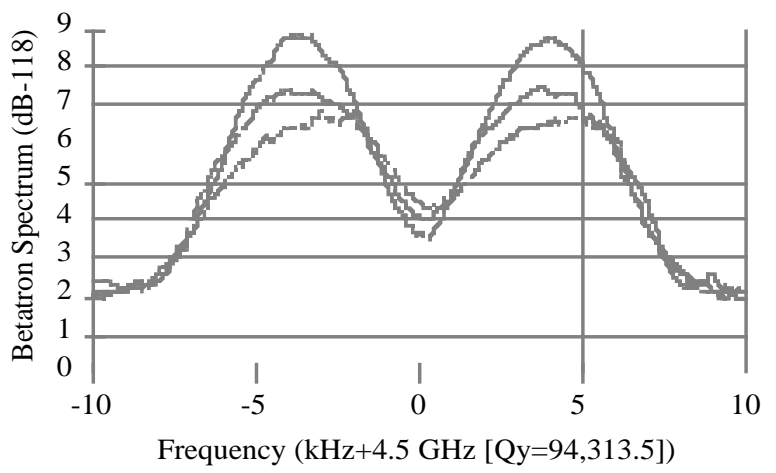

Figure 11: Measurement of signal suppression. Top, signal heating; Middle, open loop; Bottom, Signal Suppression.

\section{ACKNOWLEDGMENTS}

A large number of people have contributed their talents to this project. Although cooling of high energy bunched beams has not yet been successful, many spin-offs from the developed technology have been used in other accelerator systems at Fermilab. The pickup/kicker electrode design is the brain child of Dave McGinnis with the expert help of Pat Hurh, Jim Budlong, and Don Poll. The "super hybrid" was designed by Jeff Petter. The optical technician extraordinaire was Ernie Buchanan.
Microwave systems and support were provided by the ace team of Ken Fullett, Dave Peterson, Wes Mueller, Pete Seifrid, Pat Sheahan, and Bob Vargo. John Marriner for his stochastic cooling expertise. Last but not least was the guidance of Gerry Jackson who started this project as a Wilson Fellow.

\section{REFERENCES}

[1] G. Jackson, "Bunched Beam Stochastic Cooling", Conf. Record IEEE PAC, San Francisco (1991), p. 25322536.

[2] J. Marriner, G. Jackson, D. McGinnis, R. J. Pasquinelli, D. Peterson, and J. Petter, "Bunched Beam Cooling in the FNAL Antiproton Accumulator", Eur. Part. Acc. Conf., Nice (1990), p. 1577.

[3] D. McGinnis, "Theory and Design of Microwave Planar Electrodes for Stochastic Cooling of Particle Beams", Microwave and Optical Technology Letters, October 1991, Vol. 4/ Num. 11, p. 439-443.

[4] J. Petter, "Planar Slot Coupled Microwave Hybrid", Patent \#5,075,647; Dec. 24, 1991. CA.

[5] Ortel Corporation, 5515B optical link, Alhambra,

[6] R. J. Pasquinelli, W Kells, D. Peterson, "Optical Correlator Notch Filters for Fermilab Debuncher Betatron Stochastic Cooling", Proc. IEEE PAC, Chicago (1989), p. 694-696.

[7] Sumitomo Electric Industries, Thermally Stabilized Single Mode Fibers, Yokohama, Japan.

[8] R. J. Pasquinelli, "Electro-Optical Technology Applied to Accelerator Beam Measurement and Control", Proc. IEEE PAC, Washington DC, (1993), p. 2081-2085.

[9] BT\&D Technologies, SOA3200 Polarization Insensitive Optical Amplifier, Wilmington, DE.

[10] Channel Industries Inc., C5400 piezo cylinder, Santa Barbara, CA.

[11] Private communications with Fritz Caspers. CERN.

[12] G. Jackson, G. W. Foster, "A Storage Ring for Enhanced Antiproton Production at Fermilab", Proc, this conference IEEE PAC, Dallas, TX (1995). 